\title{
Fitting procedure based on Differential Evolution to evaluate impedance parameters of metal-coating systems*
}

\author{
Francisco González ${ }^{\dagger,}$, David Greiner ${ }^{a}$, Vicente Mena ${ }^{b}$, Ricardo M. Souto ${ }^{c, d}$, Juan \\ J. Santana ${ }^{\mathrm{b}}$, and Juan J. Aznárez ${ }^{\mathrm{a}}$ \\ ${ }^{a}$ Instituto Universitario de Sistemas Inteligentes y Aplicaciones Numéricas en \\ Ingeniería (SIANI), Universidad de Las Palmas de Gran Canaria, Spain \\ ${ }^{\mathrm{b}}$ Departamento de Ingeniería de Procesos, Universidad de Las Palmas de Gran \\ Canaria, Spain \\ ${ }^{\mathrm{c}}$ Departamento de Química, Universidad de La Laguna, Spain \\ ${ }^{\mathrm{d}}$ Instituto Universitario de Materiales y Nanotecnología (IMN), Universidad de La \\ Laguna, Spain
}

\begin{abstract}
Purpose: Impedance data obtained by Electrochemical Impedance Spectroscopy $(E I S)$ are fitted to a relevant electrical equivalent circuit in order to evaluate parameters directly related to the resistance and the durability of metal-coating systems. The purpose of this study is to present a novel and more efficient computational strategy for the modelling of EIS measurements using the differential evolution paradigm.

Design/methodology/approach: An alternative method to non-linear regression algorithms for the analysis of measured data in terms of equivalent circuit parameters is provided by Evolutionary Algorithms (EAs), particularly the Differential Evolution $(D E)$ algorithms (standard $D E$ and a representative of the self-adaptive $D E$ paradigm were used).

Findings: The results obtained with $D E$ algorithms were compared with those yielding from commercial fitting software achieving a more accurate solution, and a better parameters identification, in all the cases treated. Further, an enhanced fitting power for the modelling of metal-coating systems was obtained.

Originality/value: The great potential of the developed tool has been demonstrated in the analysis of the evolution of EIS spectra due to progressive degradation of metal-coating systems. Open codes of the different differential algorithms used are included, also examples tackled in the document are open. It allows the complete use, or improvement, of the developed tool by researchers.
\end{abstract}

Keywords: metal-coating systems, Electrochemical Impedance Spectroscopy, electrical equivalent circuit parameters, modelling studies, Evolutionary Algorithms, Differential Evolution

${ }^{*}$ This is the peer reviewed version of the following article: F. González, D. Greiner, V. Mena, R. M. Souto, J. J. Santana, J. J. Aznárez, Fitting procedure based on Differential Evolution to evaluate impedance parameters of metal-coating systems, Engineering Computations, 36(9):2960-2982, 2019, which has been published in final form at https://doi.org/10.1108/EC-11-2018-0513. This article may be used for non-commercial purposes in accordance with Emerald Terms and Conditions for the Author Accepted Manuscripts (Creative Commons Attribution Non-commercial International Licence 4.0 (CC BY-NC 4.0)).

${ }^{\dagger}$ Corresponding author: Francisco González, Instituto Universitario de Sistemas Inteligentes y Aplicaciones Numéricas en Ingeniería, Universidad de Las Palmas de Gran Canaria, Edificio Central del Parque Científico y Tecnológico del Campus Universitario de Tafira, 35017 Las Palmas de Gran Canaria, Spain.

Email: francisco.gonzalez@ulpgc.es 


\section{Introduction}

Corrosion is a natural phenomenon which converts a refined metal to a more chemically-stable form, such as its oxide, hydroxide or sulphide. It takes place in various environments causing metal components to fail and to deteriorate at different rates based on the metal composition and environmental factors such as temperature, humidity and salinity among others. The global cost of corrosion is estimated to be US $\$ 2.5$ trillion in the U.S.A., equivalent to roughly $3.4 \%$ of the global Gross Domestic Product (GDP) (NACE International 2016). With this economic impact, that is comparable to other countries, it is obvious that the fight against corrosion is necessary. A growing number of research studies are dedicated to investigate the corrosion process in several mediums and to examine possible mitigation options. Indeed, total elimination of the corrosion process may not be possible.

The most important type of corrosion process is of electrochemical nature. In this process, anodic and cathodic reactions are involved. The most typical reactions are:

Anodic, corrosion process; Metal (Me):

$$
\mathrm{Me} \longrightarrow \mathrm{Me}^{n+}+n e^{-}
$$

Cathodic, neutral or basic medium:

$$
\frac{1}{2} \mathrm{O}_{2}+\mathrm{H}_{2} \mathrm{O}+2 e^{-} \longrightarrow 2 \mathrm{OH}^{-}
$$

Acidic medium:

$$
2 \mathrm{H}^{+}+2 e^{-} \longrightarrow \mathrm{H}_{2}
$$

In order to prevent a corrosion process, one of the semi-reactions showed above has to be avoided. One of the corrosion protection techniques used is based in the establishment of a barrier between the metal and the environment surrounding the metal, blocking the diffusion of contaminants and humidity from the atmosphere to the metal surface. One of the most important techniques are organic coatings (Kendig \& Mills 2017) used to extend the life span of the metal in different environments. In particular, the easiness of application, low cost and the remarkable corrosion protection efficiency of protective coatings initiates further investigation and utilization of these techniques for corrosion protection purposes. The performance of the different organic coatings is often established on the basis of atmospheric exposure (Cambier et al. 2014, Prosek et al. 2012) and accelerated laboratory tests (Prosek et al. 2012, Cambier \& Frankel 2014). Due to the electrochemical nature of the corrosion process, techniques as EIS (Electrochemical Impedance Spectroscopy) (Cambier \& Frankel 2014, Amirudin \& Thierry 1995, Mansfeld 1995, Bonora et al. 1996, Scully \& Hensley 1994, Bierwagen et al. 2003, González-Guzmán et al. 2010) have come to be employed successfully in monitoring the physicochemical changes occurring during the exposure of a metal-coating system to a given corrosive environment, typically sea water $(\mathrm{NaCl} 3.5 \% \mathrm{w} / \mathrm{w})$. EIS technique has proved to be very useful for the detection of pores and defects in the coating (Santana et al. 2012) at significantly earlier times (Amirudin \& Thierry 1995, Kittel et al. 2001, Scully 1989, Kendig et al. 1993). Indeed, relevant information regarding both the electrochemical behaviour of the metal-coating system and the extent of the unprotected metallic areas can be extracted from the analysis of the impedance spectra.

For an EIS study, typically, painted probes are placed in an electrochemical flat cell as depicted in Figure 1, with the coated side facing the test solution acting as the working electrode. The experimental setup is completed with a reference electrode and a counter electrode. With this 
arrangement, the response evolution of the alternating current/potential (impedance $Z$ ) of the paint system can be monitored as a function of frequency. For experimental data, the frequency range usually covers the $10 \mathrm{kHz}-0.1 \mathrm{~Hz}$, with a $10 \mathrm{mV}$ amplitude for the sinusoidal voltage in order to consider the system as pseudo-linear. Due to this wide range of frequencies, it is possible to obtain information related to interfacial electrochemical phenomena (Orazem \& Tribollet 2008, Lvovich 2012) through this technique. Therefore, these data are usually fitted to an equivalent electric circuit through the use of some specific software. The analysis of the electrical parameters of this equivalent circuit, and its evolution in time, allows qualitative and quantitative characterization of the studied electrochemical system.

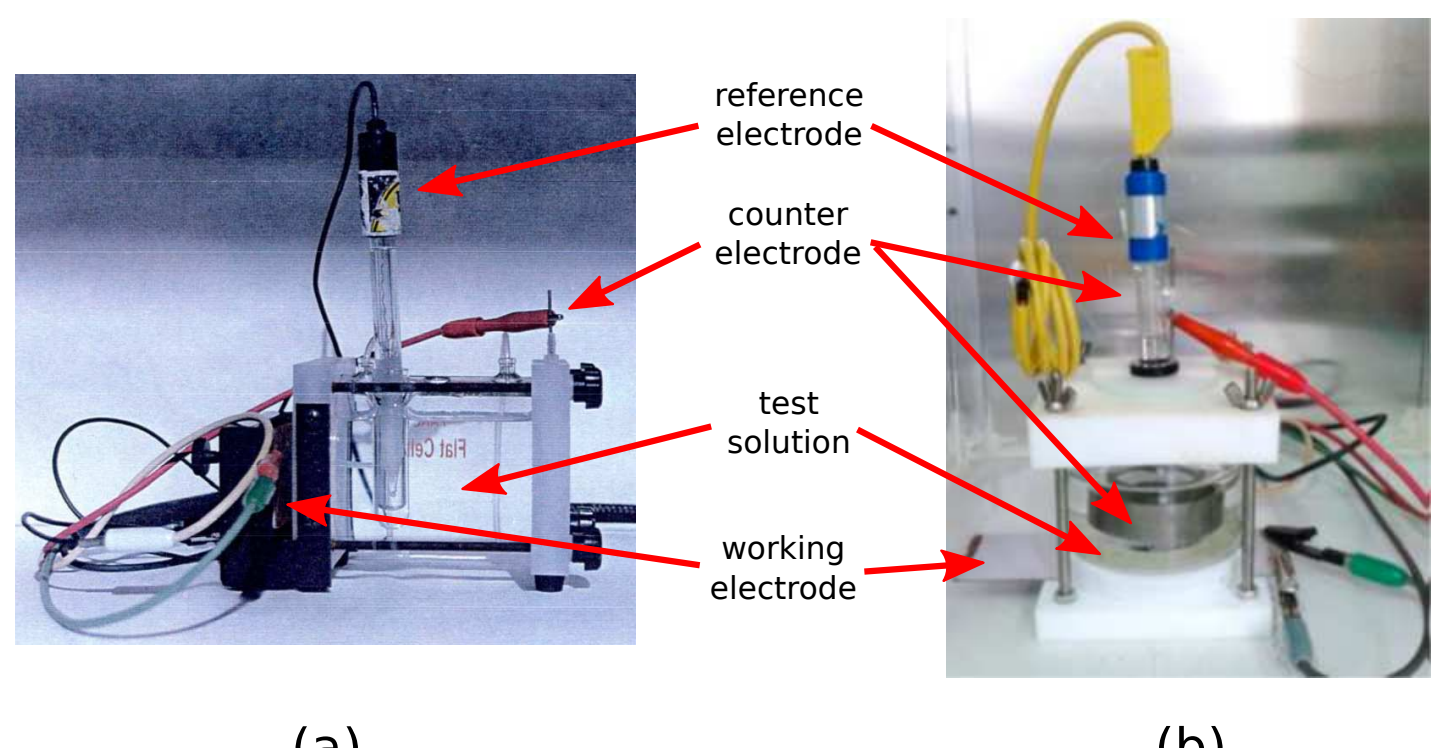

(a)

(b)

Figure 1: Typical electrochemical flat cells used for impedance measurements. The area of the sample exposed to the test electrolyte was established in the lateral of the cell (a) or at the bottom (b) by using rubber o-rings and a rigid Teflon holder to guarantee the water tightness of the system. The electrochemical setup is completed with the reference and counter electrodes. The test solution often is a $\mathrm{NaCl} 3.5 \% \mathrm{w} / \mathrm{w}$ aqueous solution, open to air, held at room temperature. Pictures: (a) taken from (Santana 1999), (b) taken specifically for the present work.

Even today, the analysis and interpretation of EIS data is an arduous task, though there are several commercial software packages available for the modelling of the experimental data, such as EQUIVCRT developed by Boukamp (Boukamp 1986), ZSimpWin (Yeum 2001), as well as other included in the software of acquisition of experimental data such as NOVA (Metrohm Autolab 2015). The adjustment procedures of the experimental data are always based on non-linear regression algorithms such as the Gauss-Newton method $(G N)$, its modified variant $(G N M)$, and the LevenbergMarquardt method ( $L M$ ) (Macdonald \& Garber 1977, Macdonald et al. 1982, Macdonald 1990, Hansen et al. 2015). The resulting fits can be very accurate if a good initial estimation of the adjustment parameters is introduced. This feature is precisely their main disadvantage or limitation, so it is often hard for these algorithms to converge to the minimum (Ratkowsky 1983). In case of existence of various local minima, the algorithm can stagnate in one of them. According to the usual procedure to avoid this situation, several executions taken from different initial points should be done so as to find the so-called good initial estimate. Although these programs can make fairly adequate initial estimates of the parameters, this does not guarantee avoidance of the aforementioned limitation. This problem has not been solved to full satisfaction.

A major development in computational optimization tools has occurred in recent years, leading to the availability of Evolutionary Algorithms (EAs) (Simon 2013, Bäck 1996, Greiner et al. 2017), which 
are new methodologies that can be applied to the analysis and adjustment of impedance data (Cao et al. 2003). The approach is inspired by the Darwinian principle of natural selection and genetic reproduction. Among the paradigms encompassed in EAs, there are genetic algorithms (Holland 1975, 1992, Goldberg 1989), genetic programming (Koza 1993) and evolution strategies (Beyer 2001), as well as Differential Evolution (DE) (Price et al. 2005). These algorithms are global optimizers due to their population search, and they can address the search for optimal single- and multi-objective solutions (Coello Coello et al. 2007). They have big advantages over traditional methods for problem optimization, because they can be applied simultaneously with integer, discontinuous or discrete variables. Furthermore, they have gained great importance for their potential as a technique for solving complex problems, and they are frequently applied in many engineering fields in general (Winter et al. 1996, Miettinen et al. 1998, Clegg \& Robinson 2012, Greiner et al. 2015, 2018), and in Process and Chemical Engineering in particular (Lucasius \& Kateman 1993, Hibbert 1993a,b, VanderNoot \& Abrahams 1998, Yu et al. 1999, Wrobel \& Miltiadou 2004, Valadi \& Siarry 2014).

Developed by Rainer Storn and Kenneth Price (Storn \& Price 1997), DE has shown good performance and quality compared to other $E A$ s in parameter adjustment problems involving real type variables analogous to the system considered in this work (Trejo-Zúñiga et al. 2013, Peng et al. 2009, Chong et al. 2012). In recent years, $D E$ has been widely used to deal with a wide range of reference tests and real-world application problems (Cai et al. 2008, Neri \& Tirronen 2010, Das \& Suganthan 2011, Rocca et al. 2011, Liu \& Qiao 2015, Saber et al. 2017). Yet, it was observed that the performance of the $D E$ search process needed to be improved due to the increasing complexity of modern optimization problems (Fan \& Zhang 2016). Among the improvements proposed to the performance of $D E$, we highlight the recent proposal of algorithms where the values of the parameters and/or operators associated with the algorithm are self-adapted during the evolution of the numerical search process. Several algorithms have been developed with these characteristics, namely, a self-adaptive $D E(j D E)$ (Brest et al. 2006), other Self-adaptive $D E$ ( $S a D E$ ) (Qin et al. 2009), Composite $D E(C o D E)$ algorithm (Wang et al. 2011), $D E$ with Dynamic Parameters Selection (DE-DPS) (Elsayed et al. 2014), an ensemble of mutation strategies and control parameters with DE (EPSDE) algorithm (Mallipeddi et al. 2011), a modified $D E$ with $p$-best crossover ( $M D E \_p B X$ ) (Islam et al. 2012), a Success-History Based Parameter Adaptation for Differential Evolution (SHADE) (Tanabe \& Fukunaga 2013), a linear population size reduction SHADE ( $L-S H A D E$ ) (Tanabe \& Fukunaga 2014), and a DE algorithm with self-adaptive strategy and control parameters (SSCPDE) (Fan \& Yan 2015).

The use of $D E$ and its variations in the chemical engineering field is going further in the recent years (Angira \& Santosh 2007, Moussa \& Awotunde 2018). Frequently, these variations are found mixed with other types of EAs as for example Genetic Algorithms GAs (Aguitoni et al. 2018). A higher amount of them are focused in the design of heat exchange networks (Babu \& Angira 2006, Zhang \& Rangaiah 2013, Aguitoni et al. 2018) rather in EIS problems like the one tackled here. A preliminary study to determine the impedance parameter setting of metal-organic coating systems modelled by equivalent electrical circuits was published by the authors in (González et al. 2015), where a standard $D E$ was applied to a problem of real impedance data corresponding to a metal-paint system (a single layer system composed of an $100 \mu \mathrm{m}$ epoxy polyamine layer applied on carbon steel plate), immersed in a solution of $\mathrm{NaCl}$. Results obtained by using standard $D E$ show improvement in the fitting quality given by commercial software. Kappel et al. (Kappel et al. 2016) compared successfully the standard $D E$ versus the Simplex method, proposing the use of experimental Direct Current values concerning the steady-state responses of an electrode as a regularization factor, besides considering EIS data. Recently, also Kappel et al. (Abud Kappel et al. 2017) used $D E$ and proposed a method for obtaining confidence regions to the estimated parameters of equivalent electrical circuits in EIS modelling with synthetic experimental data.

The main contributions of the present study are: first, to propose a fitting procedure using a more robust $D E$ algorithm approach, with a representative of the self-adaptation $D E$ capabilities, both in 
terms of operators and parameters, particularly $S a D E$ is used here in addition to the standard $D E$; second, to show how both, $D E$ or $S a D E$, give solutions even when commercial software is not able to do it; and third, to apply $D E$ algorithms both in the case of tests based on data extracted from the literature and in the case of new experimental results considering the temporal degradation of the metal-coating system. It will be shown that the fits obtained using $D E$ algorithms improve the robustness and precision of the proposed modelling solutions provided by commercial software.

The rest of the paper is structured as follows: Section 2 describes the adjustment process of electrochemical system to equivalent electrical circuit and the physical meaning of its parameters. Section 3 explains the fundamental aspects related to the $D E$ algorithm and the self-adaptive algorithm $(S a D E)$ employed in that process. Next, in Section 4, results of its application and their discussion are presented. Finally, in section 5 the main conclusions of this work are drawn.

\section{Adjustment of electrochemical impedance parameters}

For the modelling of a metal-organic coating system, it is well-known the use of an equivalent circuit that contains two time constants $R(C(R(C R))$ (Kendig \& Scully 1990, Mansfeld 1993) (see Figure 2a). It regards the polymeric coating as a non-sealing physical barrier separating the metal from the environment due to permeability of water and oxygen.

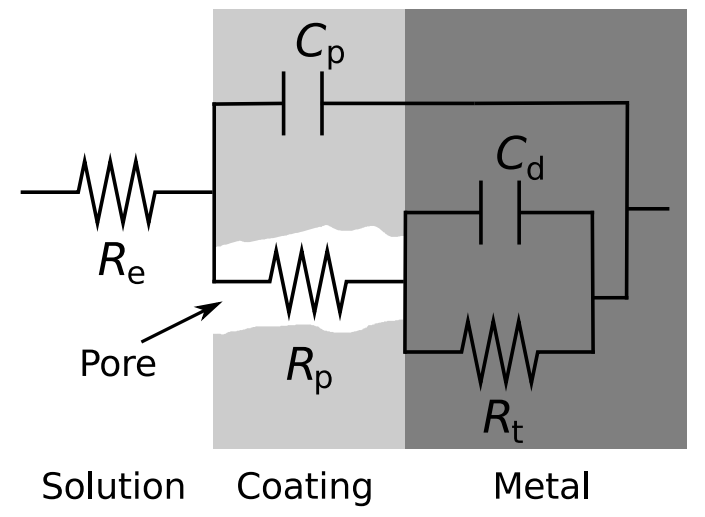

(a)

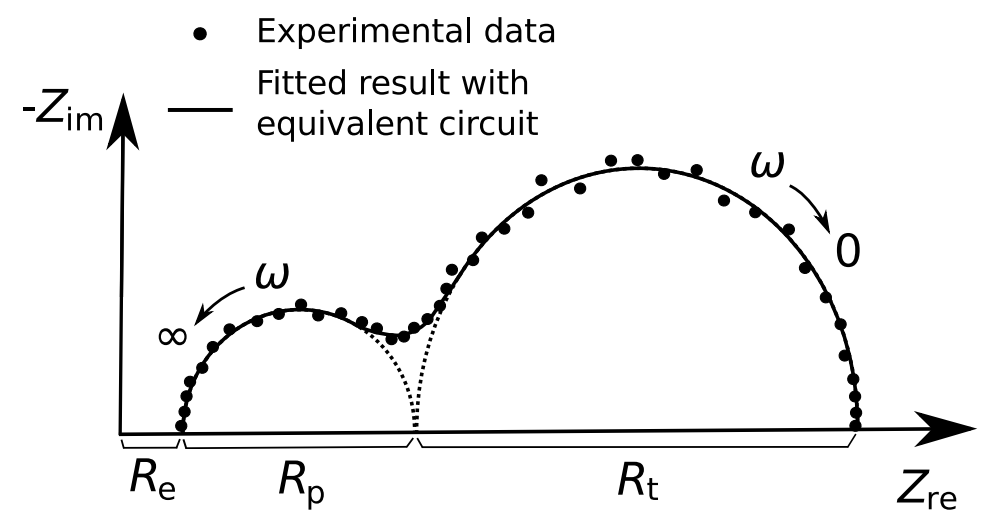

(b)

Figure 2: (a) Equivalent electric circuit used for the simulation of metal-coating system (Mansfeld 1993). (b) Typical Nyquist diagram of the metal-coating system.

In this circuit, $R_{\mathrm{e}}$ accounts for the unbalanced resistance between the reference electrode and the working electrode and remains constant all the process, $C_{\mathrm{p}}$ is the capacity of the polymer coating and $R_{\mathrm{p}}$ is the pore resistance due to the formation of conductive ion paths through the coating. $R_{\mathrm{t}}$ is the polarization resistance of the area at the metal-coating interface in which corrosion occurs, it controls the charge transfer, and $C_{\mathrm{d}}$ is the corresponding double-layer capacitance. In a typical metal-coating system under immersion in sea water $(\mathrm{NaCl} 3.5 \% \mathrm{w} / \mathrm{w})$, water will penetrate in the system with time. The coating resistance $R_{\mathrm{p}}$, which reflects the anti-penetrating ability of the coating to electrolyte solution, will decrease with time, while the coating capacitance $C_{\mathrm{p}}$, related to the diffusion behaviour of electrolyte solution in the coating, will rise with time (Kendig \& Scully 1990, Liu et al. 2009). When water and oxygen arrive at the metal interface through the coating, the electrochemical reaction site is generated at metal/electrolyte interface and corrosion process takes place.

For a satisfactory adjustment of the capacitive elements, a Constant Phase Element $(C P E)$ is usually introduced, defined as: 


$$
Z_{C P E}=\frac{1}{Q(j \omega)^{n}}
$$

where $Z_{C P E}$ is the impedance of the $C P E$ element, $j$ is the imaginary unit, $\omega$ is the angular frequency (expressed in $\mathrm{rads}^{-1}$ ), $n$ is the phase constant angle of $C P E$ (in rad), and $Q$ is the $C P E$ amplitude $\left(\mathrm{s}^{n} \Omega^{-1}\right)$. Adopting $Q$ notation for the $C P E$, the equivalent circuit adopts the form $R(Q(R(Q R))$, and its total impedance is given by:

$$
Z=R_{\mathrm{e}}+\frac{1}{Q_{\mathrm{p}}(j \omega)^{n_{\mathrm{p}}}+\frac{1}{\frac{1}{Q_{\mathrm{d}}(j \omega)^{n_{\mathrm{d}}}+\frac{1}{R_{\mathrm{t}}}}+R_{\mathrm{p}}}}
$$

Any impedance fitting procedure aims to adjust the experimental data by minimizing the error between them and the data produced by the equivalent circuit. The simplest form (and used in this work) to quantify this error, which is to be minimized, is performed through the objective function $(O F)$ written as follows:

$$
O F=\sum_{i=1}^{N}\left[\left(\overline{Z_{\mathrm{re}_{i}}}-Z_{\mathrm{re}_{i}}\right)^{2}+\left(\overline{Z_{\mathrm{im}_{i}}}-Z_{\mathrm{im}_{i}}\right)^{2}\right]
$$

where $N$ is the number of points in the experimental data corresponding to each frequency measured (number of frequencies under analysis), $\overline{Z_{\mathrm{re}_{i}}}$ and $\overline{Z_{\mathrm{im}_{i}}}$ are, respectively, real and imaginary impedance components of the adjusted values for the simulated circuit, and $Z_{\mathrm{re}_{i}}$ and $Z_{\mathrm{im}_{i}}$ the corresponding experimental values. In order to qualitatively visualize, the fit data are usually plotted as a Nyquist diagram (real component of impedance on the $x$-axis and the negative imaginary impedance component on the $y$-axis). This representation of the data is more compact and illustrative for this purpose even if the frequency dependence is not explicitly. Figure 2-b displays a typical sketch of the Nyquist diagram for the equivalent circuit of Figure 2-a.

\section{Differential Evolution algorithms}

In the following section, a description of Differential Evolution $(D E)$ and Self adaptive Differential Evolution $(S a D E)$ algorithms used in this work are presented. First, $D E$ principles and algorithm are developed in sufficient detail. Later, in the second subsection, the relevant and distinct aspects of $S a D E$ are briefly discussed.

\subsection{Standard Differential Evolution $(D E)$}

$D E$ belongs to the $E A$ s paradigm, being inspired by the natural Darwinian principle of survival of the fittest. The algorithm uses a population of $N P$ individuals ( $N P$ being the population size), which allows to optimize functions in a multidimensional continuous domain. Thus, each individual in the population is a multidimensional vector representing a possible candidate solution in the tackled optimization problem. The components of the aforementioned multidimensional vector are each of the variables of the problem (in this work: $R_{\mathrm{e}}, R_{\mathrm{p}}, R_{\mathrm{t}}, Q_{\mathrm{p}}, Q_{\mathrm{d}}, n_{\mathrm{p}}$ and $n_{\mathrm{d}}$ ). The optimum solution consists of the values of the variables obtained at the end of the optimization process. In the terminology of $E A \mathrm{~s}$, those vectors are called chromosomes, and the vector components are called genes. The chromosomes $\mathbf{x}_{i}$ are modified in a succession of iterations, where evolutionary operators, mathematical or computational, are applied on each generation.

The special feature of $D E$ is its characteristic operator of mutation, it alters the genes of a chromosome based on the idea of adding a scaled difference vector of two chosen chromosomes to a 
third one. In order to create a mutated individual $\mathbf{v}_{i}$, the selection of those chromosomes could be based on a random choice $(D E / r a n d)$ or based on the best chromosome found $(D E / b e s t)$, among other possibilities. Figure 3-a shows an example of the mutation operator in a three genes optimization problem. The parameter defining the scale factor is called $F$ hereafter. There are also versions of $D E$ with addition of more than one difference vector in the mutation operator, usually one $(D E /-/ 1$, Equation (5a)) or two $(D E /-/ 2$, Equation $(5 \mathrm{~b}))$ are the used most. This mutation operator allows variations associated to this evolution process: a) to diminish when the population is reaching the optimum solution as individuals converge to similar values; b) to adapt in every dimension (every gene) considering the higher or lower proximity to the convergence; c) to be correlated among variable dimensions which means an efficient search even in coupled problems; see, e.g., (Simon 2013).

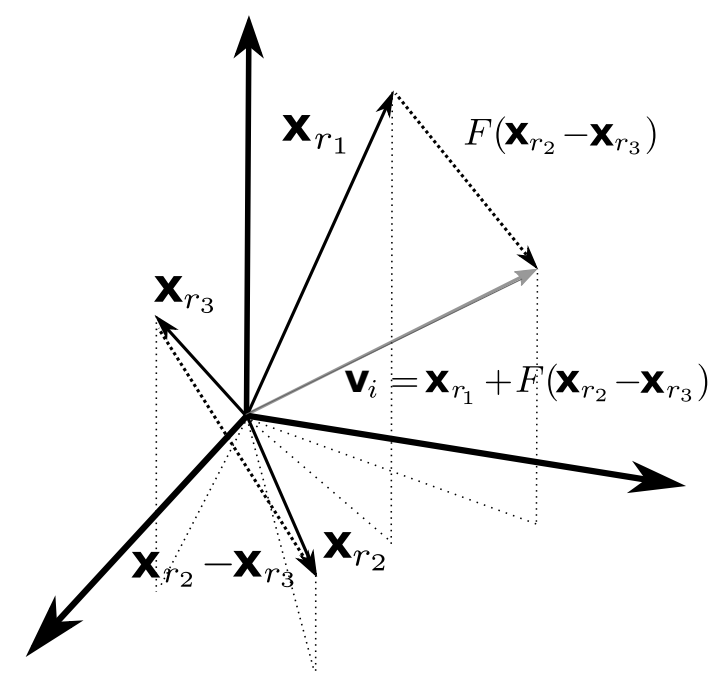

(a)

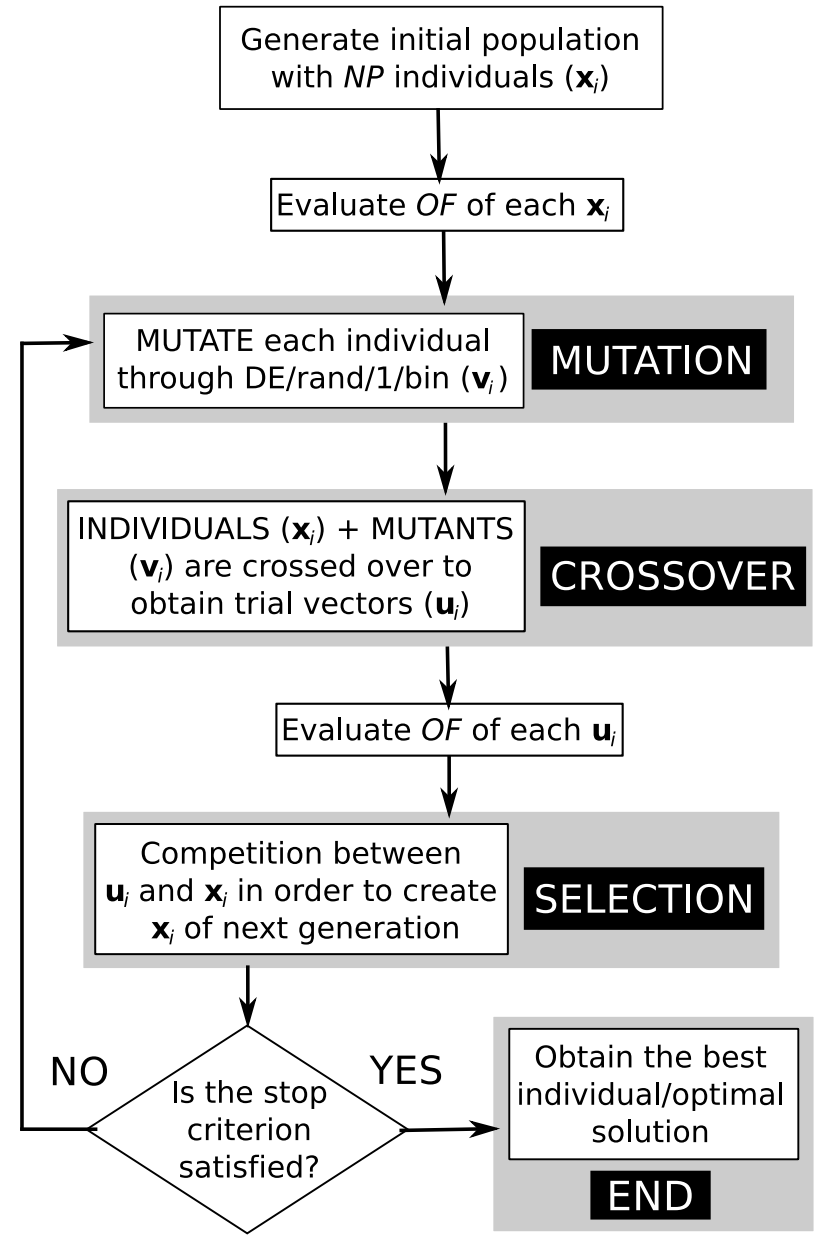

(b)

Figure 3: (a) DE mutation (three dimensional optimization problem, adapted from (Simon 2013)). (b) Block diagram of the $D E$ algorithm.

$$
\begin{array}{ll}
D E / \mathrm{rand} / 1: & \mathbf{v}_{i}=\mathbf{x}_{r 1}+F\left(\mathbf{x}_{r 2}-\mathbf{x}_{r 3}\right) \\
D E / \mathrm{rand} / 2: & \mathbf{v}_{i}=\mathbf{x}_{r 1}+F\left(\mathbf{x}_{r 2}-\mathbf{x}_{r 3}\right)+F\left(\mathbf{x}_{r 4}-\mathbf{x}_{r 5}\right)
\end{array}
$$

The crossover operator has the function of mixing the genetic information of two chromosomes to create a new individual $\mathbf{u}_{i}$. In $D E$, crossover mixes the genes of a particular chromosome $\mathbf{x}_{i}$ with a 
mutation-generated individual $\mathbf{v}_{i}$. Each gene is crossed (or not) depending on a probability variable called crossover rate $(C R)$. Binary crossover is as follows:

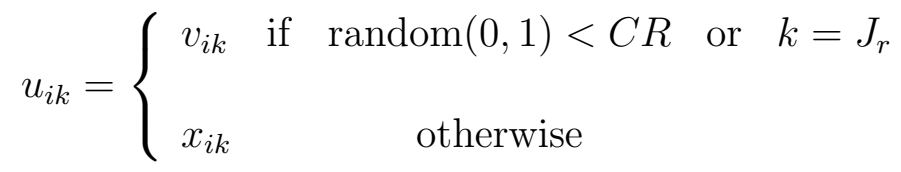

where $J_{r}$ takes an integer value between 1 and the number of genes; it guarantees that at least one component of the vector is always crossed.

Finally, the selection operator allows the fittest individuals, those with best values of the $O F$, to have more survival probabilities and therefore to propagate their genes to the following generations. The $O F$ values of the reference chromosome $\mathbf{x}_{i}$ and that generated after mutation and crossover operations $\mathbf{u}_{i}$ are compared, choosing the one with best $O F$ to be passed to the next generation and discarding the other.

The process of mutation, crossover and selection is repeated in successive generations until the stopping criterion is reached. Usually, the stopping criterion is a maximum number of generations or $O F$ evaluations that allows the population to converge upon the optimum solution.

In Figure 3-b, a diagram of the $D E$ algorithm is depicted. It starts with the creation of an initial population composed of $N P$ individuals $\mathbf{x}_{i}$. Likewise, each individual is composed by as many values as there are parameters in the equivalent electric circuit used in the model. Initially, randomly generated values are assigned to each variable being the parent population $\mathbf{x}_{i}$ in the first generation. Among the mutation alternatives, the most classical is called DE/rand/1/bin (Storn \& Price 1997). Parents $\mathbf{x}_{i}$ and mutants $\mathbf{v}_{i}$ are crossed over to generate the offspring $\mathbf{u}_{i}$, governed by the crossover rate parameter $C R$. Subsequently, offspring individuals $\mathbf{u}_{i}$ compete with their parents $\mathbf{x}_{i}$ based on their $O F$ values to decide which is transferred to the next population generation, while the loser is discarded. The parameters that govern the behaviour of the above described $D E$ algorithm are: population size $(N P)$, scale factor $(F)$ and crossover rate $(C R)$. $D E$ convergence is sensitive to the appropriate election of those parameters depending on the particular problem handled. $N P$ has a crucial role in the maintenance of equilibrium between exploration and exploitation when searching for the optimum solution, as a population with an excessive number of individuals could lead to a very slow convergence, while a population with very few individuals could lead to a premature stagnation converging to a local optimum.

\subsection{Self adaptive Differential Evolution ( $S a D E)$}

Due to its stochastic nature, to reach an appropriate performance in optimization by applying a standard $D E$ to a given problem, frequently the values of the corresponding parameters are tuned: $C R, F$ and $N P$ and the type of mutation strategy. The tuning of different parameters values and mutation strategies may affect the outcome quality depending on the robustness in a particular given problem. Even in different stages of the evolution, some values of the strategies could be more beneficial than others. Definitely, is a hard task to find the proper combination of parameters and strategies for a fast and successful convergence of the algorithm.

A $D E$ variant, which is able to self-adapt both the parameters values and mutation strategies "on the fly" according to the ongoing performance, has been implemented and tested in this work for the modelling of the EIS problem. This variant is the so-called Self adaptive Differential Evolution (SaDE) (Qin et al. 2009).

The pool of mutation strategies is as suggested by (Qin et al. 2009) and their expressions are as follows: 


$$
\begin{aligned}
\text { DE/rand/1: } & \mathbf{v}_{i}=\mathbf{x}_{r 1}+F\left(\mathbf{x}_{r 2}-\mathbf{x}_{r 3}\right) \\
D E / \text { rand/2: } & \mathbf{v}_{i}=\mathbf{x}_{r 1}+F\left(\mathbf{x}_{r 2}-\mathbf{x}_{r 3}\right)+F\left(\mathbf{x}_{r 4}-\mathbf{x}_{r 5}\right) \\
\text { DE/rand - to }- \text { best } / 2: & \mathbf{v}_{i}=\mathbf{x}_{i}+F\left(\mathbf{x}_{\text {best }}-\mathbf{x}_{i}\right)+F\left(\mathbf{x}_{r 1}-\mathbf{x}_{r 2}\right)+F\left(\mathbf{x}_{r 3}-\mathbf{x}_{r 4}\right) \\
D E / \text { current - to }- \text { rand/1: } & \mathbf{v}_{i}=\mathbf{x}_{i}+F\left(\mathbf{x}_{r 1}-\mathbf{x}_{i}\right)+F\left(\mathbf{x}_{r 2}-\mathbf{x}_{r 3}\right)
\end{aligned}
$$

The three first mutation strategies (Equations (7a) to (7c)) are associated with a binary crossover strategy, as explained in the previous subsection. On the other hand, when last mutation strategy is chosen (Equation (7d)), no crossover operation is used.

The probability for applying a particular mutation strategy depends on its capacity for generating successful chromosomes in the previous generations. The mutation strategies that have created chromosomes with lower values of $O F$ have more probability for being chosen in the current generation. In order to calculate this probability, memories are considered to store success and failure rates within a fixed number of previous generations, this is the so-called learning period $(L P)$. According to this probability, a stochastic universal sampling (Baker 1987) is applied to assign the mutation strategy to each chromosome in the current generation.

Only $N P$ and $L P$ are user specified parameters in $S a D E$, with $C R$ and $F$ being defined by normal distributions, whose initial mean value (Qin et al. 2009) is 0.5 for both parameters, with a standard deviation of 0.3 for $F$ and 0.1 for $C R$. Mean value of $C R$ is adapted through the search process based on its values in successful chromosomes generated in the previous LP generations, and there is an independent calculation for each mutation strategy.

$S a D E$ algorithm eliminates the exhaustive search for the most suitable mutation strategy and its associated $C R$ parameter on each particular case. For more details about $S a D E$ algorithm and its implementation, references (Qin et al. 2009, Qin \& Suganthan 2005) should be consulted.

$E A$ s are stochastic methods which depend on an initial random population, as well as on probabilistic based operators (mutation, crossover). Therefore, in order to compare behaviour of different parameter values, different operator types, or even different algorithms, it is necessary to compare not a single outcome of the algorithm, but a set of independent executions. In the present study, five independent runs for each algorithm were assumed.

\section{Results and discussion}

To validate the possibilities of the procedure, first, synthetic impedance data from the literature were used in order to predict impedance parameters in a system composed of two time constants (Yeum 2002, Esteban \& Orazem 1991). Next, real impedance data corresponding to the temporal degradation of a metal-coating system, immersed in a solution of $\mathrm{NaCl}$, were evaluated. It was a double layer system composed of an inner $100 \mu \mathrm{m}$ epoxy-polyamine layer and a $50 \mu \mathrm{m}$ acrylic polyurethane finishing layer applied on carbon steel plate with Sa2 1/2 surface finish. Additional details on this system can be found in (Santana et al. 2012).

A population size $(N P)$ of 200 individuals and stopping criterion of 5000 generations have been used in the $D E$ and $S a D E$ algorithms. Standard $D E$ tested with a three-parameters combination, $(F=0.5 ; C R=0.5),(F=0.9 ; C R=0.1)$ and $(F=0.1 ; C R=0.5)$, is shown. Initial mean values and the standard deviations of the Gaussian normal distribution in $S a D E$ (mean, standard deviation) were as follows: $C R=(0.5,0.1), F=(0.5,0.3)$, and learning period $(L P)$ was set to 30 generations. 


\subsection{Case 1. Validation of the procedure. TestB system taken from (Yeum 2002, Esteban \& Orazem 1991)}

The demonstration case is labelled as TestB in the ZSimpWin software (Yeum 2002), it corresponds to the adjustment of synthetic impedance data generated from the equivalent circuit in Figure 2-a to data extracted from a study performed by Esteban and Orazem (Esteban \& Orazem 1991).

Table 1 shows the values of the parameters from the equivalent circuit obtained using $D E$ and SaDE algorithms, together with those estimated using ZSimpWin (Yeum 2001) for reference. The values of the objective function $O F$ (Equation (4)) obtained in each case are also listed. The corresponding Nyquist diagrams are depicted in Figure 4-a, allowing a qualitative comparison of the experimental data with the simulated data delivered by the three fitting procedures under consideration. Additionally, Table 2 lists the final $O F$ values obtained for each of the five executions employed for $D E$ and $S a D E$, together with the average, best, worst, median and standard deviation found. The evolution of convergence using $D E$ and $S a D E$ algorithms on this system is given in Figure 4-b, whereas the horizontal line in the plot gives the corresponding $O F$ value obtained using the circuit parameters values produced by ZSimpWin. It depicts the average of best OF values (on axis $Y$ ) for each generation (on axis $X$ ). Each execution involves 48 and $50 \mathrm{~s}$ computation costs for $D E$ and $S a D E$, respectively, on an Intel Core i5-6198DE-2.30GHz computer with 8 GB RAM and 64-bit operative system.

\begin{tabular}{l|c|cc|cc} 
& ZSimp Win & \multicolumn{2}{|c|}{ DE } & \multicolumn{2}{c}{ SaDE } \\
\hline$R_{\mathrm{e}} / \Omega \mathrm{cm}^{2}$ & 9.92 & 8.66 & $(-12.70 \%)$ & 8.60 & $(-13.31 \%)$ \\
$R_{\mathrm{p}} / \Omega \mathrm{cm}^{2}$ & 111.42 & 120.70 & $(+8.33 \%)$ & 120.95 & $(+8.55 \%)$ \\
$R_{\mathrm{t}} / \Omega \mathrm{cm}^{2}$ & 190.70 & 180.88 & $(-5.15 \%)$ & 180.66 & $(-5.26 \%)$ \\
$Q_{\mathrm{p}} / \mathrm{s}^{n} \Omega^{-1} \mathrm{~cm}^{-2}$ & $1.235 \cdot 10^{-5}$ & $2.299 \cdot 10^{-5}$ & $(+86.15 \%)$ & $2.321 \cdot 10^{-5}$ & $(+87.94 \%)$ \\
$n_{\mathrm{p}}$ & 0.9758 & 0.8872 & $(-9.08 \%)$ & 0.8857 & $(-9.23 \%)$ \\
$Q_{\mathrm{d}} / \mathrm{s}^{n} \Omega^{-1} \mathrm{~cm}^{-2}$ & $2.867 \cdot 10^{-3}$ & $2.988 \cdot 10^{-3}$ & $(+4.22 \%)$ & $2.992 \cdot 10^{-3}$ & $(+4.36 \%)$ \\
$n_{\mathrm{d}}$ & 0.8474 & 0.9272 & $(+9.42 \%)$ & 0.9281 & $(+9.52 \%)$ \\
\hline $\boldsymbol{O F}$ & $\mathbf{1 . 8 1 6 4} \cdot \mathbf{1 0}^{\mathbf{3}}$ & $\mathbf{9 . 0 9 5 5 \cdot \mathbf { 1 0 } ^ { \mathbf { 2 } }}$ & $\mathbf{( - 4 9 . 9 3 \% )}$ & $\mathbf{9 . 0 9 8 2 \cdot \mathbf { 1 0 } ^ { \mathbf { 2 } }}$ & $\mathbf{( - 4 9 . 9 1 \% )}$
\end{tabular}

Table 1: Case 1. Validation of the procedure. TestB system taken from (Yeum 2002, Esteban \& Orazem 1991). Parameters of the equivalent circuit obtained with ZSimpWin (Yeum 2001), and the best solutions with $D E$ and $S a D E$ algorithms.

From the aforementioned, the following observations regarding fit operation can be extracted:

- As shown in Table 2, the obtained results with the standard $D E$ depend on the values of $F$ and $C R$ parameters. For example, the difference of the average between the best $(F=0.5$; $C R=0.5)$ and worst $(F=0.1 ; C R=0.5)$ tested parameter combination is greater than $400 \%$. The goodness of the performance of the values depends on the handled problem, and it is a priori unknown. This is a conventional behaviour of the EAs and metaheuristics ( $D E$ in our case): their parameters could greatly influence the search behaviour. On the contrary, in the more robust $S a D E$ algorithm there are no initial $F$ and $C R$ parameters to tune, and its self-adapting capabilities are able to propose a good final optimum solution, in the same order of magnitude as the best $D E$ shown in Table 2.

- According to Tables 1 and 2, the best final solution produced by among all combinations of $F$ and $C R$ parameters of $D E$ and $S a D E$ algorithms is in the same order of magnitude, both only differ on the fourth significant figure $\left(O F_{D E}=909.55\right.$ vs $\left.O F_{S a D E}=909.82\right)$. Also the average convergence of $S a D E$ is faster in the early stages of the evolution (cf. Figure 4-b) because it 

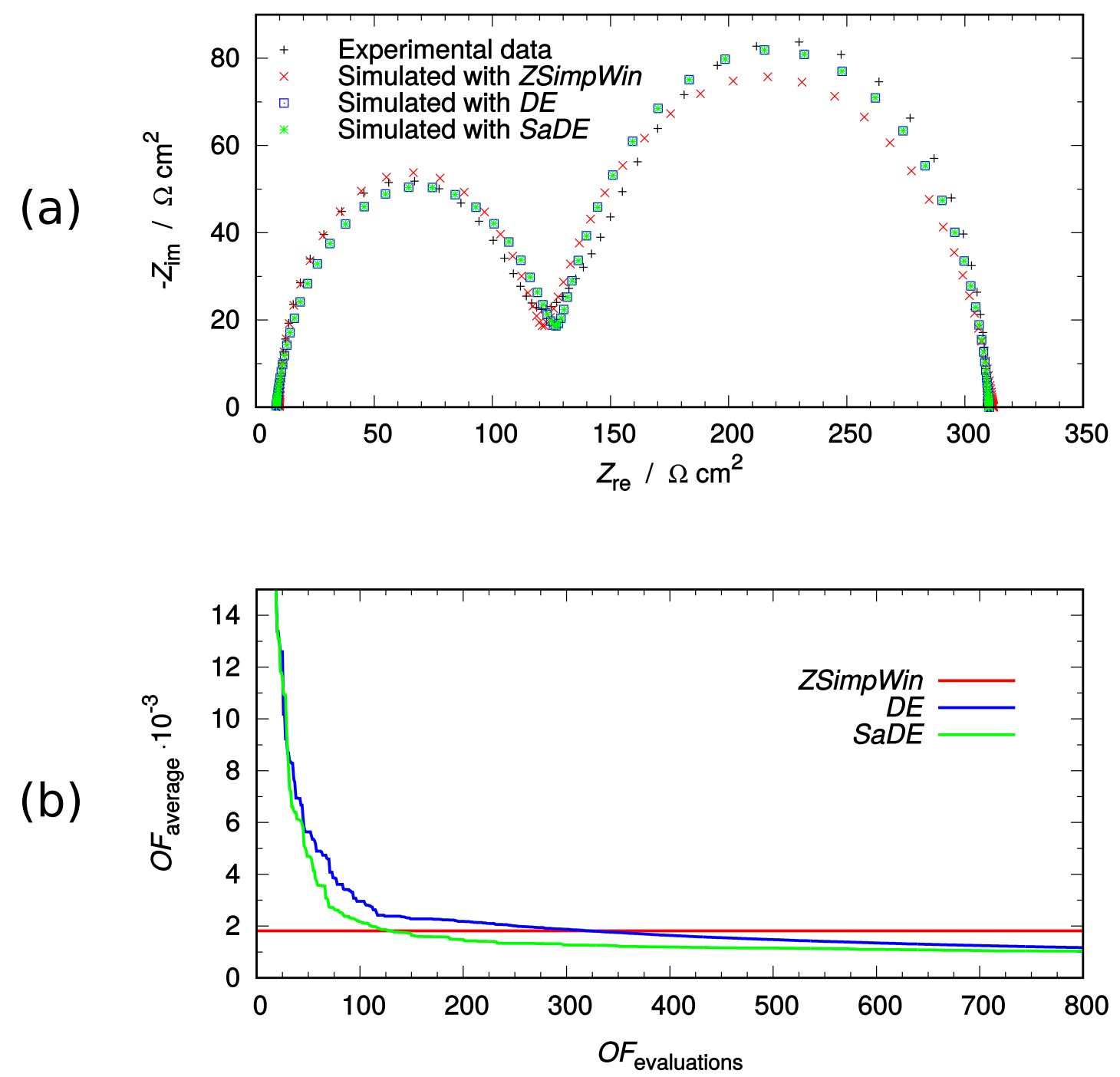

Figure 4: Case 1. Validation of the procedure. TestB system taken from (Yeum 2002, Esteban \& Orazem 1991). (a) Nyquist diagrams of experimental data and simulations to equivalent circuits obtained from the best fit with ZSimp Win (Yeum 2001) and the algorithms $D E(F=0.5 ; C R=0.5)$ and $S a D E$ used. (b) Evolution of the average convergence of best values of $O F$ obtained in each generation using $D E(F=0.5 ; C R=0.5)$ and $S a D E$ (in the interest of clarity, only the evolution in the first 800 generations is shown).

is able to select, on each stage of the evolution, the proper mutation strategy from the pool (Equation (7)) and the $F$ and $C R$ parameters combination.

- There is a significant improvement of the best fitted plots using $D E$ algorithms compared to that delivered by the commercial software taken for comparison purposes. Thus, the objective function given by ZSimp Win amounted $O F=1.8164 \cdot 10^{3}$, whereas smaller values (in the order of $50 \%)$ are obtained using either $D E\left(O F=9.0955 \cdot 10^{2}\right)$ or $S a D E\left(O F=9.0982 \cdot 10^{2}\right)$ algorithms. The differences between the circuit parameters values obtained by using $D E$ or $S a D E$, and the values obtained by using ZSimpWin depend on the efficiency of the optimization method and the parameter sensitivity in the model. This differences are between $4 \%$ and $14 \%$ for $R_{\mathrm{e}}, R_{\mathrm{p}}$, $R_{\mathrm{t}}, n_{\mathrm{p}}, Q_{\mathrm{d}}$ and $n_{\mathrm{d}}$, and is almost $90 \%$ for $Q_{\mathrm{p}}$ (cf. Table 1 ). Thus, in this problem, although in some parameters, the value differences are maintained in a low range, in others, these can be very high. All this suggests that, while $D E$ algorithms have found the global optimum, ZSimp Win has been probably trapped in a local suboptimum. 


\begin{tabular}{|c|c|c|c|c|c|c|c|}
\hline & \multicolumn{2}{|c|}{$D E$} & \multicolumn{2}{|c|}{$D E$} & \multicolumn{2}{|c|}{$D E$} & \multirow[b]{3}{*}{$S a D E$} \\
\hline & $F$ & $C R$ & $F$ & $C R$ & $F$ & $C R$ & \\
\hline & 0.9 & 0.1 & 0.1 & 0.5 & 0.5 & 0.5 & \\
\hline Execution 1 & \multicolumn{2}{|c|}{1145.5034887} & \multicolumn{2}{|c|}{2614.7362320} & \multicolumn{2}{|c|}{909.5516225} & 909.8188502 \\
\hline Execution 2 & \multicolumn{2}{|c|}{1082.5334186} & \multicolumn{2}{|c|}{6051.9642576} & \multicolumn{2}{|c|}{909.5512448} & 911.4402325 \\
\hline Execution 3 & \multicolumn{2}{|c|}{971.1022822} & \multicolumn{2}{|c|}{2786.0926525} & \multicolumn{2}{|c|}{909.5521927} & 916.0706725 \\
\hline Execution 4 & \multicolumn{2}{|c|}{967.2706802} & \multicolumn{2}{|c|}{3360.1016781} & \multicolumn{2}{|c|}{909.5513893} & 927.2312285 \\
\hline Execution 5 & \multicolumn{2}{|c|}{997.6611296} & \multicolumn{2}{|c|}{5392.5337059} & \multicolumn{2}{|c|}{909.5511607} & 911.2602060 \\
\hline Average & \multicolumn{2}{|c|}{1032.8141999} & \multicolumn{2}{|c|}{4041.0857052} & \multicolumn{2}{|c|}{909.5515220} & 915.1642379 \\
\hline Best & \multicolumn{2}{|c|}{967.2706802} & \multicolumn{2}{|c|}{2614.7362320} & \multicolumn{2}{|c|}{909.5511607} & 909.8188502 \\
\hline Worst & \multicolumn{2}{|c|}{1145.5034887} & \multicolumn{2}{|c|}{6051.9642576} & \multicolumn{2}{|c|}{909.5521927} & 927.2312285 \\
\hline Median & \multicolumn{2}{|c|}{997.6611296} & \multicolumn{2}{|c|}{3360.1016781} & \multicolumn{2}{|c|}{909.5513893} & 911.4402325 \\
\hline Standard deviation & \multicolumn{2}{|c|}{70.02} & \multicolumn{2}{|c|}{1410.20} & \multicolumn{2}{|c|}{$3.70 \cdot 10^{-4}$} & 6.39 \\
\hline
\end{tabular}

Table 2: Case 1. Validation of the procedure. TestB system taken from (Yeum 2002, Esteban \& Orazem 1991). OF statistics of $D E$ and $S a D E$ algorithms executions for the experimental data shown in Figure 4-a.

\subsection{Case 2. Temporal degradation of a metal-coating system composed of two layers epoxy-polyamine $(100 \mu \mathbf{m})+$ acrylic polyurethane $(50 \mu \mathbf{m})$ coating on carbon steel plate (Santana et al. 2012)}

Simulations were also performed on a two-layer coating system applied on a carbon steel plate with Sa2 1/2 surface finish. In brief, the inner coating layer was a $100 \mu \mathrm{m}$ thick epoxy-polyamine primer, and the topcoat consisted in $50 \mu \mathrm{m}$ thick polyurethane. Impedance spectra were recorded at different times up to $890 \mathrm{~h}$ during exposure in $\mathrm{NaCl} 3.0 \% \mathrm{w} / \mathrm{w}$ aqueous solution, by applying a $10 \mathrm{mV}$ amplitude sinusoidal voltage in the $10 \leq f \leq 50 \mathrm{kHz}$ frequency range, 14 points per decade (Santana et al. 2012). A flat three-electrode configuration was employed, with the coated metal placed at the bottom of the cell facing upwards to the solution, with $5.56 \mathrm{~cm}^{2}$ exposed area.

In previous case, $S a D E$ was shown to be a more robust option than $D E$; this, joined with the similar outcomes provided by both algorithms (once the proper combination of $C R$ and $F$ parameters for $D E$ algorithm was found), are the reasons why only $S a D E$ is considered here. Here, a population size $(N P)$ of 200 individuals, stopping criterion of 10000 iterations and learning period $(L P)$ of 30 generations have been used.

Figure 5 shows a comparison, in a typical Nyquist graph, between the experimental data corresponding to measurements at various elapsed times, namely, 150, 215, 310, 385, 480, 550, 650, 720 and $890 \mathrm{~h}$, and the simulations obtained using ZSimpWin and SaDE. It should be noticed that no convergence was obtained using ZSimpWin for some of the exposures (215, 385, 480, 720 and 890 h), whereas the experimental data could always be fitted using $S a D E$. The normalized $O F$ values corresponding to the optimal solutions obtained for the five programmed executions of the $S a D E$ algorithm to each impedance spectrum are depicted in Figure 6. Among them, the execution that offers the best fit on each elapsed time, which have been joined using a black line, was employed to produce the simulated spectra in Figure 5. The total calculation time required to perform each of these independent runs was $55 \mathrm{~s}$ on an Intel Core i5-6198DE-2.30GHz computer with 8 GB RAM and 64-bit operating system. Similar execution time than Case 1 is obtained for a double number of iterations because the number of points of experimental data are almost half.

From the aforementioned, the following observations regarding fit operation can be extracted:

- The use of the $S a D E$ algorithm allowed fitting the chosen equivalent circuit to all the experimental impedance spectra, whereas ZSimpWin failed to find a consistent solution in some 

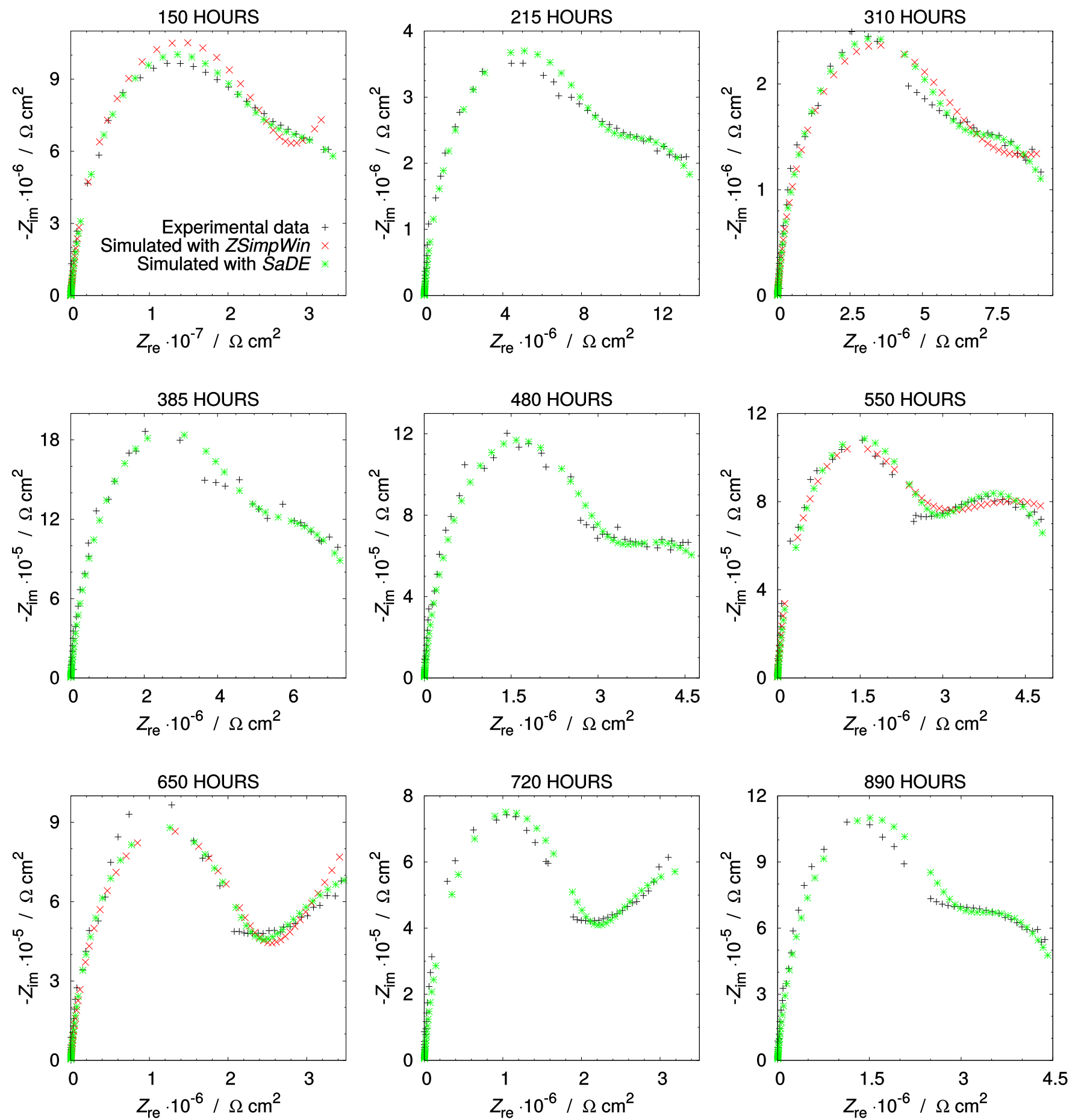

Figure 5: Case 2. Temporal degradation of a metal-coating system composed of two layers epoxypolyamine $(100 \mu \mathrm{m})+$ acrylic polyurethane $(50 \mu \mathrm{m})$ coating on carbon steel plate (Santana et al. 2012). Evolution of the Nyquist diagram. Experimental data and response of equivalent circuits obtained using ZSimpWin (Yeum 2001) and SaDE algorithm. 


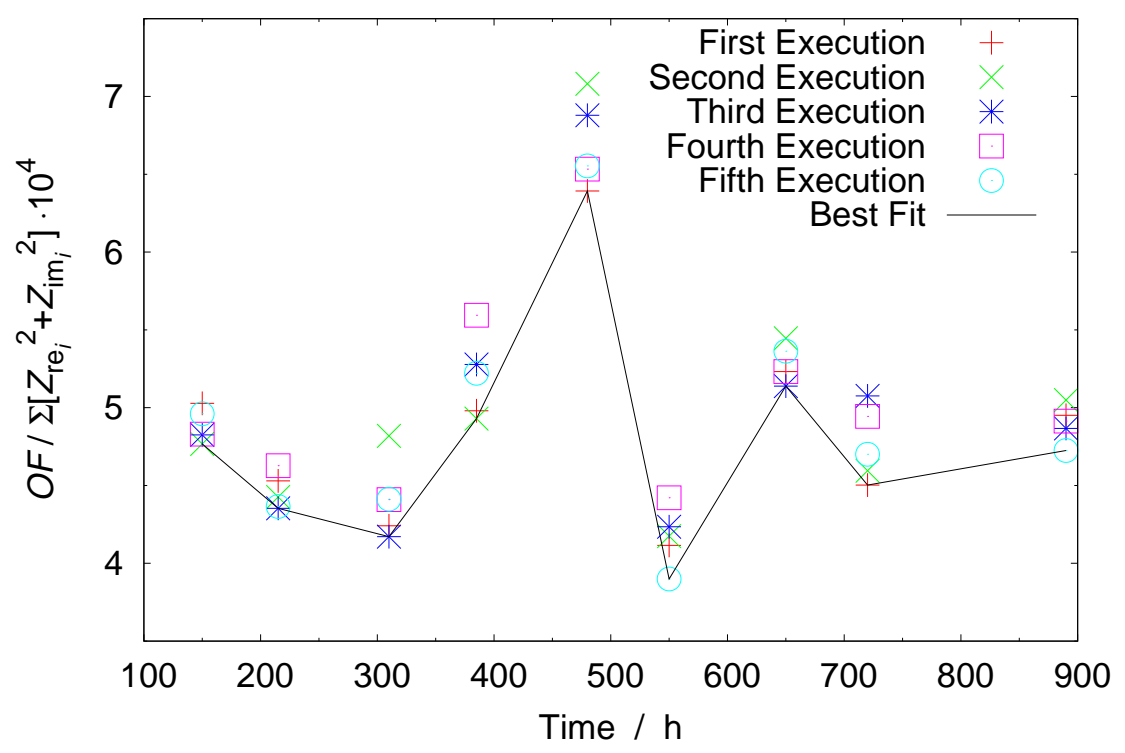

Figure 6: Case 2. Temporal degradation of a metal-coating system composed of two layers epoxypolyamine $(100 \mu \mathrm{m})+$ acrylic polyurethane $(50 \mu \mathrm{m})$ coating on carbon steel plate (Santana et al. 2012). Normalized values of $O F$ for the 5 executions carried out with the proposed $S a D E$ algorithm.

cases. In order to obtain a solution with ZSimpWin, one of the possibilities carried out by chemical engineers is through a two steps procedure. First, experimental data are fitted by using a equivalent circuit constituted by pure capacitors instead of $C P E$ s, i.e. a $R(C(R(C R))$ equivalent circuit. Obtained parameters values are used as a seed in the second step of the fitting procedure with the target equivalent circuit, $R(Q(R(Q R))$. This way, but not in all cases, a solution can be obtained using ZSimpWin for experimental EIS data which were not able to be fitted directly with the $R(Q(R(Q R))$ equivalent circuit. This procedure is not necessary when using $S a D E$, which provides a proper fitting to all the experimental impedance spectra.

- Only small variations are found among the fits obtained in the five executions considered (see Figure 6), a feature also observed in the system used for verification in Case 1. It demonstrates again the robustness of the $S a D E$ algorithm.

- Though the Nyquist plots may significantly vary for measurements done at different elapsed times in the test solution, the $O F$ values for the five executions exhibit similar variations for each impedance spectrum. Therefore, the $S a D E$ algorithm is able to find a solution for the equivalent circuit in a robust and reliable way and, above all, continuously over the temporal degradation of the system.

Relevant information regarding both the electrochemical behaviour of the metal-coating system and the extent of the corrosion process can be extracted from the analysis of the impedance spectra for different immersion times. The temporal analysis of the different parameters offer a good idea about the performance of the metal-coating system. These parameters are plotted as a function of the duration of exposure for the metal-coating system under investigation in Figure 7, allowing the evolution of the process to be monitored. The time degradation of fitted circuit components is consistent with the typical expectations for a metal-coating system. The coating resistance $R_{\mathrm{p}}$ exhibits a steady decay with the elapse of time, amounting to one order of magnitude for the duration of the experiment (i.e, a diminution from initial values close to $2.5 \cdot 10^{7} \Omega \mathrm{cm}^{2}$ down to $2.5 \cdot 10^{6} \Omega \mathrm{cm}^{2}$ after $890 \mathrm{~h}$ ), as it is logical, being subjected to an aggressive environment such as a salt water solution. That is, ionic pathways are progressively developed through the coating allowing the underlying metal 
to be exposed to the environment. Next, the component $R_{\mathrm{t}}$, related to the charge transfer resistance at the metal-coating interface, shows an almost 10 -fold decrease (namely from $1.6 \cdot 10^{7} \Omega \mathrm{cm}^{2}$ to $2.0 \cdot 10^{6} \Omega \mathrm{cm}^{2}$ ), indicating that the corrosion rate of the metal increases simultaneously to the loss of barrier characteristics of the polymer coating. In turn, areas where corrosion has started it extends, and therefore, the corrosion resistance decreases. On the other hand, $Q_{\mathrm{p}}$ and $Q_{\mathrm{d}}$ increase due to the water infiltration in the coating and in the metal-coating interface, respectively.

\section{Conclusions}

This paper presents a novel computational strategy for the modelling of Electrochemical Impedance Spectroscopy $(E I S)$ measurements using a Self-adaptive Differential Evolution algorithm $(S a D E)$.

The applicability of the new procedure has been tested in metal-coating systems exposed to aqueous electrolyte solutions that present two time constants in their impedance spectra, usually described by an equivalent circuit of the type $R(Q(R(Q R))$.

The $S a D E$ algorithm was implemented and tested both with a set of synthetic impedance data taken from the literature, and with a real metal-coating system composed of a $100 \mu \mathrm{m}$ epoxypolyamine layer and a $50 \mu \mathrm{m}$ acrylic polyurethane finishing layer applied on carbon steel plate. The results obtained in this work are very promising, because they significantly improve those delivered by the simulation and adjustment of the same systems using the commercial software most widely employed by scientists working on the characterization of metal-coating systems (namely ZSimp Win).

It is well known that some unusual EIS data hardly yield good fitting results by non-linear regression algorithms. In addition, to get a solution provided by the commercial software was no possible for some cases tackled in this work.

Work in progress focuses on the application of this numerical method to the physicochemical characterization of other electrochemical systems, as well as to improve the efficiency of the developed algorithm. In any case, the scientific objective of this first phase of the work has been to demonstrate the suitability of the methodology to deal with the problem taking into account two requirements: low computing time and the simplicity in the control parameters of the algorithm ( $S a D E$ only has three parameters to be tuned). The first result of the work has been an efficient, accurate (more accurate and stable than the available commercial software) and easy-to-use tool for professionals who are not specialist in computational engineering.

Further research is needed to see if better results can be achieved, and the possibility is completely open for continuing in the close future by comparing among different self-adapted $D E$, in addition to $S a D E$.

\section{Acknowledgements}

This work was supported by the Agencia Canaria de Investigación, Innovación y Sociedad de la Información (ACIISI) of the Government of Canary Islands (Spain) and FEDER through research project ProID2017010042. F. González is a recipient of the fellowship FPU14/06936 from the Ministerio de Educación, Cultura y Deporte of Spain. The authors are grateful for this support. 

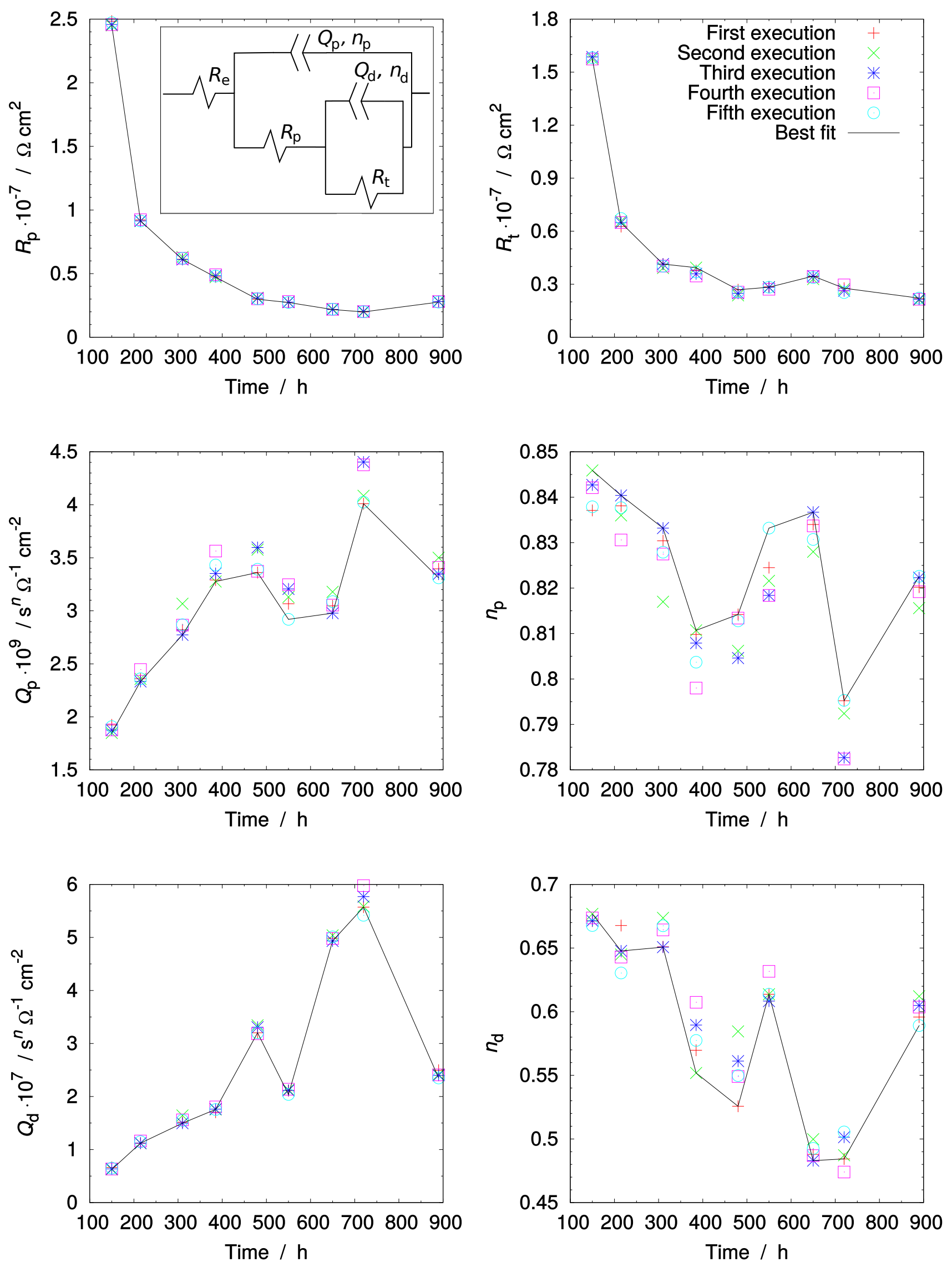

Figure 7: Case 2. Temporal degradation of a metal-coating system composed of two layers epoxypolyamine $(100 \mu \mathrm{m})+$ acrylic polyurethane $(50 \mu \mathrm{m})$ coating on carbon steel plate (Santana et al. 2012). Evolution of the parameters of the equivalent circuit obtained from the $S a D E$ setting. 


\section{Supplementary material}

Supplementary material associated with this article can be found at DOI: 10.5281/zenodo.2654862. On the compressed file, the open codes in $\mathrm{C}++$ language for $D E$ and $S a D E$ algorithms are included on the corresponding folders prepared for compiling with GNU compiler (Stallman 2012). Examples of input and output files for each algorithm are also included. The call graph between functions are depicted on Figure 8 for each algorithm, (a) $D E$ and (b) $S a D E$.

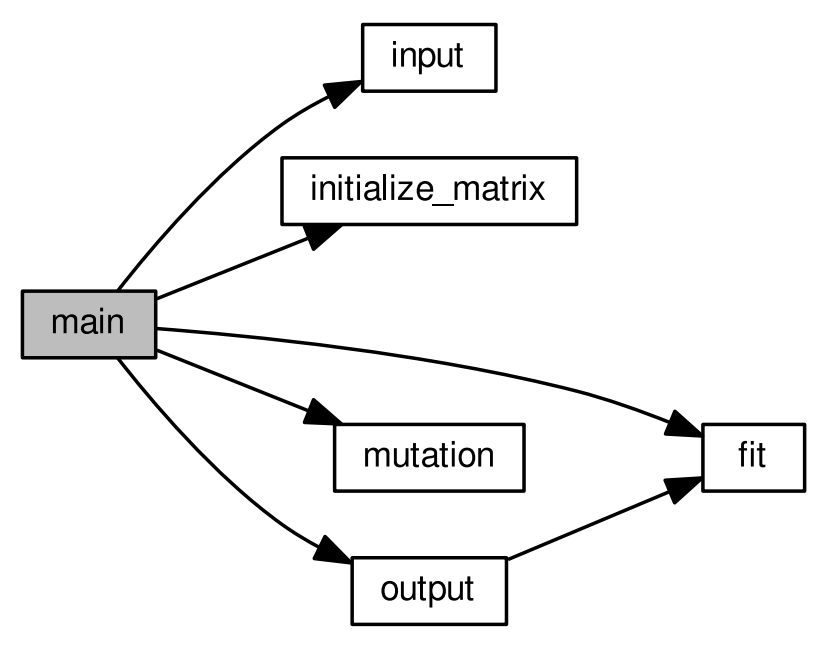

(a)

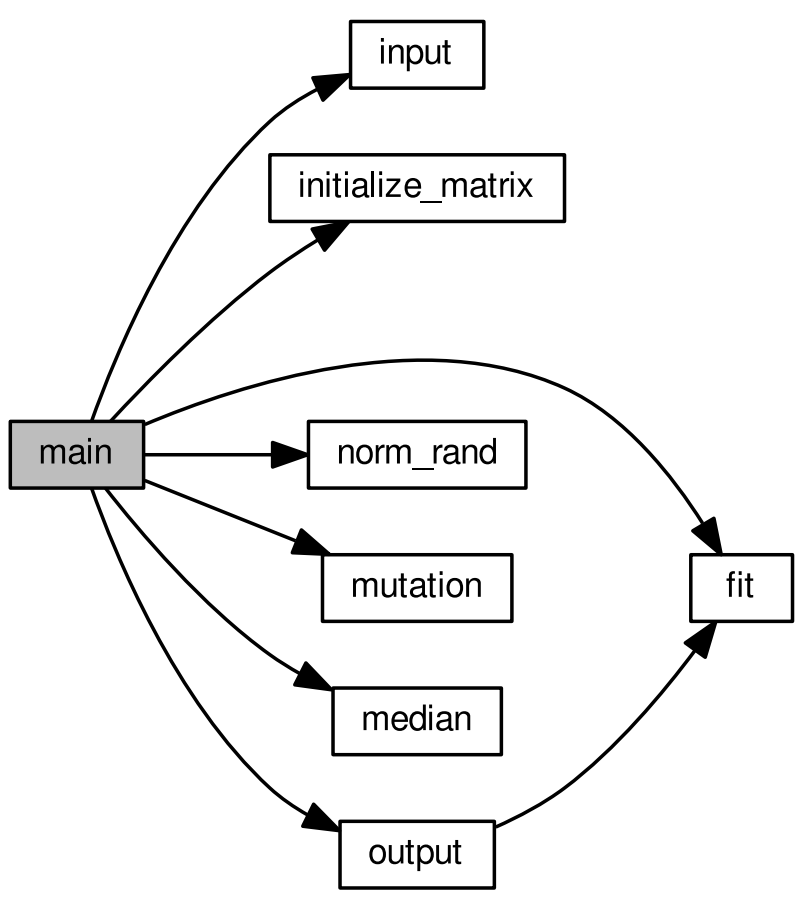

(b)

Figure 8: Call graph between algorithm functions. (a) DE and (b) SaDE.

On the other hand, executable files and simulation examples included in this work are also included in the compressed file, and structured according to the cases (Sections 4.1 and 4.2, respectively).

\section{References}

Abud Kappel, M. A., Peixoto, F. C., Platt, G. M., Domingos, R. P. \& Bastos, I. N. (2017), 'A study of equivalent electrical circuit fitting to electrochemical impedance using a stochastic method', Appl. Soft Comput. 50, 183-193. doi: $10.1016 /$ j.asoc.2016.11.030.

Aguitoni, M. C., Pavão, L. V., Siqueira, P. H., Jiménez, L. \& da Silva Sá Ravagnani, M. A. (2018), 'Heat exchanger network synthesis using genetic algorithm and differential evolution', Comput. Chem. Eng. 117, 82-96. doi: 10.1016/j.compchemeng.2018.06.005.

Amirudin, A. \& Thierry, D. (1995), 'Application of electrochemical impedance spectroscopy to study the degradation of polymer-coated metals', Prog. Org. Coat. 26(1), 1-28. doi: 10.1016/0300-9440(95)00581-1.

Angira, R. \& Santosh, A. (2007), 'Optimization of dynamic systems: A trigonometric differential evolution approach', Comput. Chem. Eng. 31(9), 1055-1063. doi: 10.1016/j.compchemeng.2006.09.015.

Babu, B. V. \& Angira, R. (2006), 'Modified differential evolution (MDE) for optimization of non-linear chemical processes', Comput. Chem. Eng. 30(6), 989-1002. doi: 10.1016/j.compchemeng.2005.12.020. 
Bäck, T. (1996), Evolutionary Algorithms in Theory and Practice: Evolution Strategies, Evolutionary Programming, Genetic Algorithms, Oxford University Press.

Baker, J. E. (1987), Reducing Bias and Inefficiency in the Selection Algorithm, in 'Proceedings of the Second International Conference on Genetic Algorithms and Their Application’, Hillsdale, NJ, USA, pp. 14-21.

Beyer, H.-G. (2001), The Theory of Evolution Strategies, Natural Computing Series, Springer-Verlag Berlin Heidelberg. doi: $10.1007 / 978-3-662-04378-3$.

Bierwagen, G., Tallman, D., Li, J., He, L. \& Jeffcoate, C. (2003), 'EIS studies of coated metals in accelerated exposure', Prog. Org. Coat. 46(2), 149-158. doi: 10.1016/S0300-9440(02)00222-9.

Bonora, P. L., Deflorian, F. \& Fedrizzi, L. (1996), 'Electrochemical impedance spectroscopy as a tool for investigating underpaint corrosion', Electrochim. Acta 41(7-8), 1073-1082. doi: 10.1016/0013-4686(95)00440-8.

Boukamp, B. A. (1986), 'A Nonlinear Least Squares Fit procedure for analysis of immittance data of electrochemical systems', Solid State Ion. 20(1), 31-44. doi: 10.1016/0167-2738(86)90031-7.

Brest, J., Greiner, S., Boskovic, B., Mernik, M. \& Zumer, V. (2006), 'Self-Adapting Control Parameters in Differential Evolution: A Comparative Study on Numerical Benchmark Problems', IEEE Trans. Evol. Comput. 10(6), 646-657. doi: 10.1109/TEVC.2006.872133.

Cai, H. R., Chung, C. Y. \& Wong, K. P. (2008), 'Application of Differential Evolution Algorithm for Transient Stability Constrained Optimal Power Flow', Comput. Sci. Inform. Syst. 23(2), 719-728. doi: 10.1109/TPWRS.2008.919241.

Cambier, S. M. \& Frankel, G. S. (2014), 'Coating and Interface Degradation of Coated steel, Part 2: Accelerated Laboratory Tests', Electrochim. Acta 136, 442-449. doi: 10.1016/j.electacta.2014.05.111.

Cambier, S. M., Posner, R. \& Frankel, G. S. (2014), 'Coating and interface degradation of coated steel, Part 1: Field exposure', Electrochim. Acta 133, 30-39. doi: 10.1016/j.electacta.2014.04.004.

Cao, H., Yu, J. \& Kang, L. K. (2003), An evolutionary approach for modeling the equivalent circuit for electrochemical impedance spectroscopy, in 'The 2003 Congress on Evolutionary Computation', Vol. 3, Canberra, ACT, Australia, pp. 1819-1825. doi: 10.1109/CEC.2003.1299893.

Chong, C. K., Mohamad, M. S., Deris, S., Shamsir, S., Abdullah, A., Choon, Y. W., Chai, L. E. \& Omatu, S. (2012), Using an Improved Differential Evolution Algorithm for Parameter Estimation to Simulate Glycolysis Pathway, in S. Omatu, J. De Paz Santana, S. González, J. Molina, A. Bernardos \& J. Rodríguez, eds, 'Distributed Computing and Artificial Intelligence. Advances in Intelligent and Soft Computing', Vol. 151, Springer-Verlag Berlin Heidelberg, pp. 709-716. doi: 10.1007/978-3-642-28765-7_85.

Clegg, J. \& Robinson, M. P. (2012), 'A genetic algorithm for optimizing multi-pole Debye models of tissue dielectric properties', Phys. Med. Biol. 57(19), 6227-6243. doi: 10.1088/0031-9155/57/19/6227.

Coello Coello, C. A., Lamont, G. B. \& van Veldhuizen, D. A. (2007), Evolutionary Algorithms for Solving MultiObjective Problems, Genetic and Evolutionary Computation Series, Springer US. doi: 10.1007/978-0-387-36797-2.

Das, S. \& Suganthan, P. N. (2011), 'Differential Evolution: A Survey of the State-of-the-Art', IEEE Trans. Evol. Comput. 15(1), 4-31. doi: 10.1109/TEVC.2010.2059031.

Elsayed, S. M., Sarker, R. A. \& Essam, D. L. (2014), 'A self-adaptive combined strategies algorithm for constrained optimization using differential evolution', Appl. Math. Comput. 241, 267-282. doi: 10.1016/j.amc.2014.05.018.

Esteban, J. M. \& Orazem, M. E. (1991), 'On the Application of the Kramers-Kronig Relations to Evaluate the Consistency of Electrochemical Impedance Data', J. Electrochem. Soc. 138(1), 67-76. doi: 10.1149/1.2085580.

Fan, Q. \& Yan, X. (2015), 'Differential evolution algorithm with self-adaptive strategy and control parameters for P-xylene oxidation process optimization', Soft Comput. 19(5), 1363-1391. doi: 10.1007/s00500-014-1349-y.

Fan, Q. \& Zhang, Y. (2016), 'Self-adaptive differential evolution algorithm with crossover strategies adaptation and its application in parameter estimation', Chemom. Intell. Lab. Syst. 151, $164-171 . \quad$ doi: 10.1016/j.chemolab.2015.12.020.

Goldberg, D. E. (1989), Genetic Algorithms in Search, Optimization and Machine Learning, Addison-Wesley. 
González, F., Greiner, D., Aznárez, J. J., Mena, V., Souto, R. M. \& Santana, J. J. (2015), 'Chemical process simulation using evolutionary algorithms: application to the analysis of impedance parameters of electrochemical systems (in Spanish)', Afinidad 72(572), 278-283.

González-Guzmán, J., Santana, J. J., González, S. \& Souto, R. M. (2010), 'Resistance of metallic substrates protected by an organic coating containing glass flakes', Prog. Org. Coat. 68(3), 240-243. doi: 10.1016/j.porgcoat.2010.01.005.

Greiner, D., Galván, B., Périaux, J., Gauger, N., Giannakoglou, K. \& Winter, G., eds (2015), Advances in Evolutionary and Deterministic Methods for Design, Optimization and Control in Engineering and Sciences, Vol. 36 of Computational Methods in Applied Sciences, Springer International Publishing. doi: 10.1007/978-3-319-11541-2.

Greiner, D., Periaux, J., Emperador, J. M., Galván, B. \& Winter, G. (2017), 'Game Theory Based Evolutionary Algorithms: A Review with Nash Applications in Structural Engineering Optimization Problems', Arch. Comput. Methods Eng. 24(4), 703-750. doi: 10.1007/s11831-016-9187-y.

Greiner, D., Periaux, J., Quagliarella, D., Magalhães-Mendes, J. \& Galván, B. (2018), 'Evolutionary Algorithms and Metaheuristics: Applications in Engineering Design and Optimization', Math. Probl. Eng. 2018(Article ID 2793762), 1-4. doi: 10.1155/2018/2793762.

Hansen, J. K., Hogue, J. D., Sander, G. K., Renaut, R. A. \& Popat, S. C. (2015), 'Non-negatively constrained least squares and parameter choice by the residual periodogram for the inversion of electrochemical impedance spectroscopy data', J. Comput. Appl. Math. 278, 52-74. doi: 10.1016/j.cam.2014.09.017.

Hibbert, D. B. (1993a), 'A hybrid genetic algorithm for the estimation of kinetic parameters', Chemom. Intell. Lab. Syst. 19(3), 319-329. doi: 10.1016/0169-7439(93)80031-C.

Hibbert, D. B. (1993b), 'Genetic algorithms in chemistry', Chemom. Intell. Lab. Syst. 19(3), $277-293$. doi: 10.1016/0169-7439(93)80028-G.

Holland, J. H. (1975), Adaptation in Natural and Artificial Systems: An introductory analysis with applications to biology, control, and artificial intelligence, University of Michigan Press.

Holland, J. H. (1992), 'Genetic Algorithms', Sci. Amer. 267(1), 66-72. doi: 10.1038/scientificamerican0792-66.

Islam, S. M., Das, S., Ghosh, S., Roy, S. \& Suganthan, P. N. (2012), 'An Adaptive Differential Evolution Algorithm With Novel Mutation and Crossover Strategies for Global Numerical Optimization', IEEE Trans. Syst. Man Cybern. B Cybern. 42(2), 482-500. doi: 10.1109/TSMCB.2011.2167966.

Kappel, M. A., Fabbri, R., Domingos, R. P. \& Bastos, I. N. (2016), 'Novel electrochemical impedance simulation design via stochastic algorithms for fitting equivalent circuits', Meas. 94, 344-354. doi: 10.1016/j.measurement.2016.08.008.

Kendig, M., Jeanjaquet, S. \& Lumsden, J. (1993), Electrochemical Impedance of Coated Metal Undergoing Loss of Adhesion, in J. R. Scully, D. C. Silverman \& M. W. Kendig, eds, 'Electrochemical Impedance. Analysis and Interpretation', Vol. STP1188, American Society for Testing and Materials, pp. 407-427. doi: 10.1520/STP18082S.

Kendig, M. \& Mills, D. J. (2017), 'An historical perspective on the corrosion protection by paints', Prog. Org. Coat. 102(A), 53-59. doi: 10.1016/j.porgcoat.2016.04.044.

Kendig, M. \& Scully, J. (1990), 'Basic Aspects of Electrochemical Impedance Application for the Life Prediction of Organic Coatings on Metals', Corros. 46(1), 22-29. doi: 10.5006/1.3585061.

Kittel, J., Celati, N., Keddam, M. \& Takenouti, H. (2001), 'New methods for the study of organic coatings by EIS: New insights into attached and free films', Prog. Org. Coat. 41(1-3), 93-98. doi: 10.1016/S0300-9440(00)00155-7.

Koza, J. R. (1993), Genetic programming: on the programming of computers by natural selection. Complex Adaptive Systems Series, Vol. 1, MIT Press.

Liu, J. \& Qiao, S. (2015), 'A image segmentation algorithm based on differential evolution particle swarm optimization fuzzy c-means clustering', Comput. Sci. Inform. Syst. 12(2), 873-893. doi: 10.2298/CSIS141108031L.

Liu, X., Xiong, J., Lv, Y. \& Zuo, Y. (2009), 'Study on corrosion electrochemical behavior of several different coating systems by EIS', Prog. Org. Coat. 64(4), 497-503. doi: 10.1016/j.porgcoat.2008.08.012.

Lucasius, C. B. \& Kateman, G. (1993), 'Understanding and using genetic algorithms Part 1. Concepts, properties and context', Chemom. Intell. Lab. Syst. 19(1), 1-33. doi: 10.1016/0169-7439(93)80079-W. 
Lvovich, V. F. (2012), Impedance epectroscopy: applications to electrochemical and dielectric phenomena, John Wiley \& Sons, Inc. doi: 10.1002/9781118164075.

Macdonald, J. R. (1990), 'Impedance spectroscopy: old problems and new developments', Electrochim. Acta 35(10), 1483-1492. doi: 10.1016/0013-4686(90)80002-6.

Macdonald, J. R. \& Garber, J. A. (1977), 'Analysis of Impedance and Admittance Data for Solids and Liquids', J. Electrochem. Soc. 124(7), 1022-1030. doi: 10.1149/1.2133473.

Macdonald, J. R., Schoonman, J. \& Lehnen, A. P. (1982), 'Applicability and power of complex nonlinear least squares for the analysis of impedance and admittance data', J. Electroanal. Chem. Interfacial Electrochem. 131, 77-95. doi: 10.1016/0022-0728(82)87062-9.

Mallipeddi, R., Suganthan, P. N., Pan, Q. K. \& Tasgetiren, M. F. (2011), 'Differential evolution algorithm with ensemble of parameters and mutation strategies', Appl. Soft Comput. 11(2), 1679-1696. doi: 10.1016/j.asoc.2010.04.024.

Mansfeld, F. (1993), 'Models for the impedance behavior of protective coatings and cases of localized corrosion', Electrochim. Acta 38(14), 1891-1897. doi: 10.1016/0013-4686(93)80311-M.

Mansfeld, F. (1995), 'Use of electrochemical impedance spectroscopy for the study of corrosion protection by polymer coatings', J. Appl. Electrochem. 25(3), 187-202. doi: 10.1007/BF00262955.

Metrohm Autolab, B. V. (2015), 'NOVA Advanced Electrochemical Software'.

Miettinen, K., Neittaanmäki, P., Mäkelä, M. N. \& Périaux, J., eds (1998), Evolutionary Algorithms in Engineering and Computer Science: Recent Advances in Genetic Algorithms, Evolution Strategies, Evolutionary Programming, Genetic Programming and Industrial Applications, John Wiley \& Sons.

Moussa, T. M. \& Awotunde, A. A. (2018), 'Self-adaptive differential evolution with a novel adaptation technique and its application to optimize ES-SAGD recovery process', Comput. Chem. Eng. 118, 64-76. doi: 10.1016/j.compchemeng.2018.07.018.

NACE International (2016), 'Economic Impact - Assessment Of The Global Cost Of Corrosion'. [accessed 15 October $2018]$.

URL: http://impact.nace.org/economic-impact.aspx

Neri, F. \& Tirronen, V. (2010), 'Recent advances in differential evolution: a survey and experimental analysis', Artif. Intell. Rev. 33(1-2), 61-106. doi: 10.1007/s10462-009-9137-2.

Orazem, M. E. \& Tribollet, B. (2008), Electrochemical Impedance Spectroscopy, John Wiley \& Sons, Inc. doi: $10.1002 / 9780470381588$.

Peng, B., Liu, B., Zhang, F.-Y. \& Wang, L. (2009), 'Differential evolution algorithm-based parameter estimation for chaotic systems', Chaos Solitons Fractals 39(5), 2110-2118. doi: 10.1016/j.chaos.2007.06.084.

Price, K. V., Storn, R. M. \& Lampinen, J. A. (2005), Differential Evolution: A Practical Approach to Global Optimization, Springer-Verlag Berlin Heidelberg. doi: 10.1007/3-540-31306-0.

Prosek, T., Nazarov, A., Stoulil, J. \& Thierry, D. (2012), 'Evaluation of the tendency of coil-coated materials to blistering: Field exposure, accelerated tests and electrochemical measurements', Corros. Sci. 61, 92-100. doi: 10.1016/j.corsci.2012.04.026.

Qin, A. K., Huang, V. L. \& Suganthan, P. N. (2009), 'Differential Evolution Algorithm With Strategy Adaptation for Global Numerical Optimization', IEEE Trans. Evol. Comput. 13(2), 398-417. doi: 10.1109/TEVC.2008.927706.

Qin, A. K. \& Suganthan, P. N. (2005), Self-adaptive Differential Evolution Algorithm for Numerical Optimization, in 'IEEE Congress on Evolutionary Computation', Vol. 2, Edinburgh, Scotland, UK, pp. 1785-1791. doi: 10.1109/CEC.2005.1554904.

Ratkowsky, D. A. (1983), Nonlinear Regression Modeling: A Unified Practical Approach, Marcel Dekker.

Rocca, P., Oliveri, G. \& Massa, A. (2011), 'Differential Evolution as Applied to Electromagnetics', IEEE Antennas Propag. Mag. 53(1), 38-49. doi: 10.1109/MAP.2011.5773566. 
Saber, M. G., Ahmed, A. \& Sagor, R. H. (2017), 'Performance Analysis of a Differential Evolution Algorithm in Modeling Parameter Extraction of Optical Material', Silicon 9(5), 723-731. doi: 10.1007/s12633-016-9422-z.

Santana, J. J. (1999), Estudio de la corrosión atmosférica del acero al carbono en la provincia de Las Palmas mediante técnicas clásicas y electroquímicas. Elaboración del mapa de corrosividad (in Spanish), PhD thesis, Universidad de Las Palmas de Gran Canaria.

URL: http://hdl.handle.net/10553/1826

Santana, J. J., González, J. E., Morales, J., González, S. \& Souto, R. M. (2012), 'Evaluation of ecological organic paint coatings via electrochemical impedance spectroscopy', Int. J. Electrochem. Sci. 7(7), 6489-6500.

Scully, J. R. (1989), 'Electrochemical impedance of organic-coated steel: correlation of impedance parameters with long-term coating deterioration', J. Electrochem. Soc. 136(4), 979-990. doi: 10.1149/1.2096897.

Scully, J. R. \& Hensley, S. T. (1994), 'Lifetime prediction for organic coatings on steel and a magnesium alloy using electrochemical impedance methods', Corros. 50(9), 705-716. doi: 10.5006/1.3293547.

Simon, D. (2013), Evolutionary Optimization Algorithms, Wiley.

Stallman, R. M. (2012), Using the GNU Compiler Collection, Free Software Foundation.

Storn, R. \& Price, K. (1997), 'Differential Evolution - A Simple and Efficient Heuristic for global Optimization over Continuous Spaces', J. Global Optim. 11(4), 341-359. doi: 10.1023/A:1008202821328.

Tanabe, R. \& Fukunaga, A. (2013), Success-History Based Parameter Adaptation for Differential Evolution, in '2013 IEEE Congress on Evolutionary Computation (CEC)', Cancún, México, pp. 71-78. doi: 10.1109/CEC.2013.6557555.

Tanabe, R. \& Fukunaga, A. S. (2014), Improving the Search Performance of SHADE Using Linear Population Size Reduction, in '2014 IEEE Congress on Evolutionary Computation (CEC)', Beijing, China, pp. 1658-1665. doi: 10.1109/CEC.2014.6900380.

Trejo-Zúñiga, E. C., López-Cruz, I. L. \& Ruíz-García, A. (2013), 'Estimación de parámetros para un modelo de crecimiento de cultivos usando algoritmos evolutivos y bio-inspirados', Agrocienc. 47, 671-682.

Valadi, J. \& Siarry, P., eds (2014), Applications of Metaheuristics in Process Engineering, Springer International Publishing. doi: 10.1007/978-3-319-06508-3.

VanderNoot, T. J. \& Abrahams, I. (1998), 'The use of genetic algorithms in the non-linear regression of immittance data', J. Electroanal. Chem. 448(1), 17-23. doi: 10.1016/S0022-0728(97)00593-7.

Wang, Y., Cai, Z. \& Zhang, Q. (2011), 'Differential Evolution With Composite Trial Vector Generation Strategies and Control Parameters', IEEE Trans. Evol. Comput. 15(1), 55-66. doi: 10.1109/TEVC.2010.2087271.

Winter, G., Périaux, J., Galán, M. \& Cuesta, P. (1996), Genetic Algorithms in Engineering and Computer Science, 1st edn, John Wiley \& Sons, Inc.

Wrobel, L. C. \& Miltiadou, P. (2004), 'Genetic algorithms for inverse cathodic protection problems', Eng. An. Bound. El. 28(3), 267-277. doi: 10.1016/S0955-7997(03)00057-2.

Yeum, B. (2001), 'Electrochemical Impedance Spectroscopy: Data Analysis Software, Echem Software'.

Yeum, B. (2002), Technical Note 1: Extrapolation using Kramers-Kronig relations, Technical report, EChem Software.

Yu, J., Cao, H., Chen, Y., Kang, L. \& Yang, H. (1999), 'A new approach to the estimation of electrocrystallization parameters', J. Electroanal. Chem. 474(1), 69-73. doi: 10.1016/S0022-0728(99)00307-1.

Zhang, H. \& Rangaiah, G. P. (2013), 'One-step approach for heat exchanger network retrofitting using integrated differential evolution', Comput. Chem. Eng. 50, 92-104. doi: 10.1016/j.compchemeng.2012.10.018. 